\title{
МИГРАЦИОННАЯ МОДЕЛЬ РАССЕИВАНИЯ ПРИМЕСИ В ЗАПЫЛЕННОЙ ТУРБУЛЕНТНОЙ СПУТНОЙ СТРУЕ
}

\author{
(Представил Н. Алумяэ)
}

Предложено описание процессов рассеивания инерционной примеси в двухфазной спутной турбулентной струе и рассмотрено ее распространение. На основе миграционной и турбулентно-диффузионной моделей осуществлен анализ распределения твердой прнмеси в двухфазной турбулентной спутной струе, проведено сравнение с экспериментом.

Исследуемые двухфазные спутные струи - специфичны, а именно, однофазная чистая струя распространяется в окружающем двухфазном спутном потоке.

B $\left.{ }^{1}{ }^{1}\right]$ теоретически интегральным методом расчета с учетом модели влияния примеси на турбулентность $\left[{ }^{2}\right]$ было предсказано подавление турбулентности чистой струи частицами, попавшими из окружающего спутного двухфазного потока. И как следствие, обнаружено сужение границ и увеличение дальнобойности (уменьшается темп осевого затухания скорости) внутренней струи по сравнению с аналогичной по структуре, но однофазной чистой спутной струей. Проведенные экспериментальные исследования двухфазной турбулентной струи при двух параметрах спутности $(m=0,47$ и 1,45$\left.){ }^{[3}\right]$ подтвердили это, однако оказалось, что влияние примеси очень незначительное. Это, видимо, связано с тем, что рассматривались слабоконцентрированные потоки с расходной концентрацией не превышающей единицы $\left(x_{0} \sim 0,6-0,8\right.$ кг/кг). В ходе экспериментов были получены дополнительные результаты о распределении концентрации примеси в двухфазной спутной струе, что послужило причиной проведения более глубокого и детального исследования таких струй.

В настоящей работе двухфазные спутные струи исследовались при тех же параметрах спутности $\left(m=u_{\mathrm{g}} / u_{\mathrm{g} m_{0}}=0,47\right.$ и 1,45$)$ с расходной концентрацией $x_{0}=0,85$ и $0,65 \mathrm{\kappa r} / \mathrm{kг}$ и размером частиц примеси (бронза) $\delta=45 \cdot 10^{-6}$ и $67 \cdot 10^{-6}$ м соответственно. В первом случае скорость истечения чистого газа из внутренней трубы диаметром $d=10^{-2}$ м составляла $u_{g m 0}=60 \mathrm{~m} / \mathrm{c}$, а скорость спутного двухфазного потока из внешней коаксиальной трубы диаметром $D=2 r_{0}=3 \cdot 10^{-2}$ м составляла $u_{g}=35 \mathrm{~m} / \mathrm{c}$. Во втором случае эти скорости были соответственно $u_{g m_{0}}=$ $=24,2 \mathrm{~m} /$ с и $u_{g}=35 \mathrm{~m} / \mathrm{c}$. Режимные параметры заимствовались из [ $\left.{ }^{3}\right]$. Начальные поля массовой концентрации $\alpha$ и скоростей газовой $u_{g}$ и дисперсной $u_{s}$ фаз, а также скорости чистой однофазной спутной струи $u$ брались также из $\left[{ }^{3}\right]$ и представлены на рис. 3.

Систему уравнений двухфазной турбулентной осесимметричной струи (в безразмерной форме) после осреднения и оценки пульсационных членов можно записать в виде

$$
\frac{\partial u}{\partial x}+\frac{1}{r} \frac{\partial}{\partial r} r v=0,
$$




$$
\begin{gathered}
u \frac{\partial u}{\partial x}+v \frac{\partial u}{\partial r}=1 \frac{1}{r} \frac{\partial}{\partial r} r \varepsilon \frac{\partial u}{\partial r} \\
u \frac{\partial \varepsilon}{\partial x}+v \frac{\partial \varepsilon}{\partial r}=\frac{1}{r} \frac{\partial}{\partial r} r(\gamma \varepsilon+v) \frac{\partial \varepsilon}{\partial r}+\beta \varepsilon\left|\frac{\partial u}{\partial r}\right| \\
\frac{\partial u_{g}}{\partial x}+\frac{1}{r} \frac{\partial}{\partial r} r v_{g}=0 \\
u_{g} \frac{\partial u_{g}}{\partial x}+v_{g} \frac{\partial u_{g}}{\partial r}=\frac{1}{r} \frac{\partial}{\partial r} r \varepsilon_{g} \frac{\partial u_{g}}{\partial r}-\alpha\left(f_{D}\left(u_{g}-u_{s}\right)-f_{M}\left(v_{g}-v_{s}\right)\right) \\
u_{s} \frac{\partial \alpha}{\partial x}+v_{s} \frac{\partial \alpha}{\partial r}=\frac{1}{r} \frac{\partial}{\partial r} r D_{s} \frac{\partial \alpha}{\partial r}-\alpha\left(\frac{\partial u_{s}}{\partial x}+\frac{1}{r} \frac{\partial}{\partial r} r v_{s}\right) \\
u_{s} \frac{\partial u_{s}}{\partial x}+\left(v_{s}-\left(D_{s}+\varepsilon_{s}\right) \frac{\partial \alpha}{\alpha \partial r}\right) \frac{\partial u_{s}}{\partial r}=\frac{1}{r} \frac{\partial}{\partial r} r \varepsilon_{s} \frac{\partial u_{s}}{\partial r}+ \\
+f_{D}\left(u_{g}-u_{s}\right)-f_{M}\left(v_{g}-v_{s}\right) \\
u_{s} \frac{\partial \omega}{\partial x}+\left(v_{s}-\left(D_{s}+\varepsilon_{s}\right) \frac{\partial \alpha}{\alpha \partial r}\right) \frac{\partial \omega}{\partial r}=1 \frac{\partial}{r}+\varepsilon_{s} \frac{\partial \omega}{\partial r}-f_{\omega} \omega \\
u_{s} \frac{\partial \alpha}{\partial x}+\left(v_{s}-\frac{\partial v_{s}}{\partial r}=\frac{1}{r} \frac{\partial}{\partial r} r \varepsilon_{s} \frac{\partial v_{s}}{\partial r}+\right.
\end{gathered}
$$

Система уравнений решалась при следующих граничных условиях:

$$
\begin{gathered}
x \geqslant 0, \quad r=0, \quad \frac{\partial u}{\partial r}=\frac{\partial u_{g}}{\partial r}=\frac{\partial u_{s}}{\partial r}=\frac{\partial \alpha}{\partial r}=\frac{\partial \varepsilon}{\partial r}=0, \\
v=v_{g}=v_{s}=\omega=0 ; \\
x \geqslant 0, \quad r=\delta, \quad u_{\delta}=0,02 u_{m}, \quad u_{g 0}=0,02 u_{g m}, \quad u_{s \delta}=0,02 u_{s m}, \\
\alpha_{\delta}=0,02 \alpha_{m}, \quad \omega_{\delta}=0,02 \omega_{m}, \quad v_{s \delta}=0,02 v_{s m}, \quad \varepsilon_{\delta}=v .
\end{gathered}
$$

Обезразмеривающей величиной для координат является радиус внешней трубы $r_{0}$, для скоростей фаз - скорость газовой фазы на срезе выходного устройства в центральной точке $u_{g m 0}$, для угловой скорости частиц - комбинации $u_{g m o} / r_{0}$, для коэффициентов турбулентной и ламинарной вязкостей фаз и турбулентной диффузии - комбинация $u_{g m 0} \cdot r_{0}$. Величины $\gamma$ и $\beta$ - суть числовые постоянные, имеющие значения 7 и 0,2 соответственно.

Индексом $\delta$ отмечается асимптотическая граница внешнего и внутреннего пограничного слоя, а индексами $m, m 0$ и $0-$ значения параметров потока по осевой координате, в начальном сечении в центре струи и вообще в начальном сечении струи. Для внутренней границы пограничного слоя условие (11) принимает общепринятую форму записи спутных течений, т. е. $u_{\delta}=0,47 u_{m 0}, u_{g \delta}=0,47 u_{g m 0}$ при параметре спутности $m=0,47$ или $u_{\delta}=1,45 u_{m 0}, u_{g \delta}=1,45 u_{\text {gmo }}$ при параметре спутности $m=$ $=1,45$. При решении задачи нет необходимости использовать условие (11) для внутренней границы пограничного слоя, поскольку внешний по отношению к однофазной струе двухфазный спутный поток хотя и был много шире ядра течения (в 3 раза), он был ограничен и рассматривался 
как затопленный по отношению к внешнему неподвижному пространству, что и отражено в используемом условии (11).

Начальные распределения скоростей чистой струи и газовой фазы в двухфазной струе задавались при обонх параметрах спутности в виде «ступенек», а скорости дисперсной фазы и концентрации примеси - в виде «прямоугольной ямы» (рис. 1), что соответствует данным экспери-

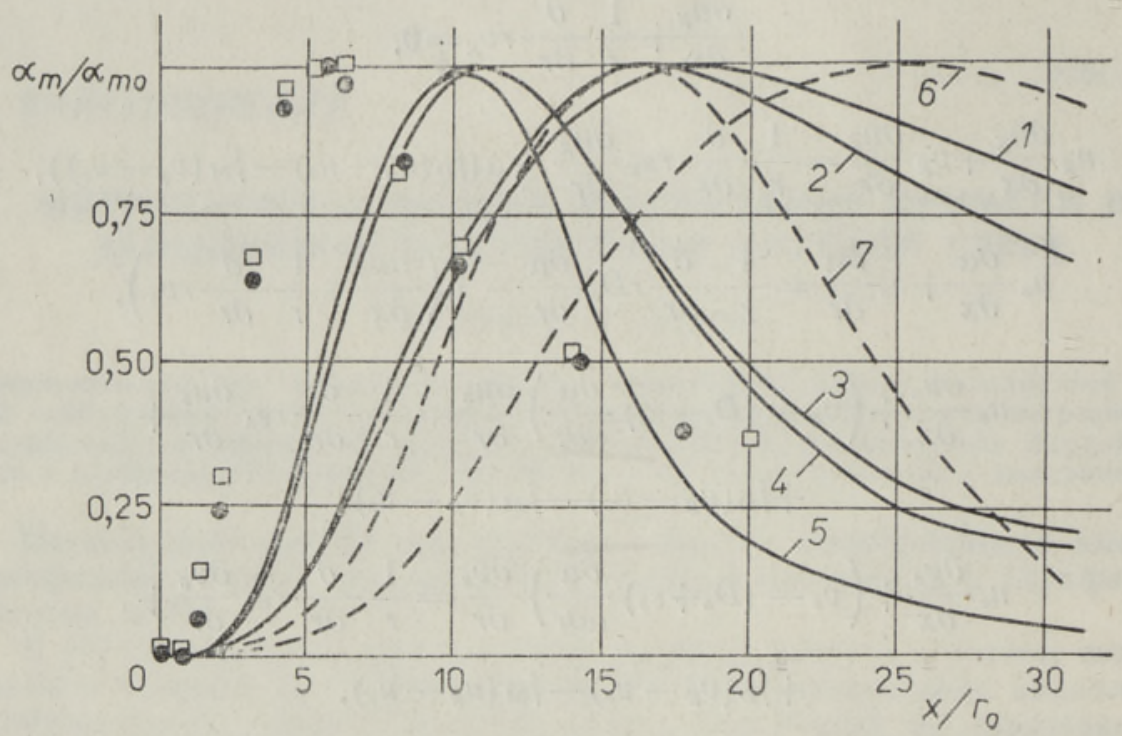

Рис. 1. Осевое распределение концентрации примеси при двух параметрах спутности:

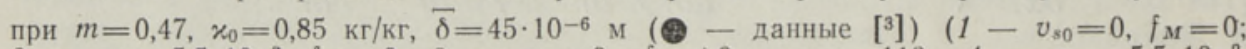
$2-v_{s 0}=5,5 \cdot 10^{-2}, f_{M}=0 ; 3-v_{s 0}=0, f_{M} \neq 0, \omega_{m 0}=-110 ; \quad 4-v_{s 0}=5,5 \cdot 10^{-2}$, $\left.f_{M} \neq 0, \omega_{m 0}=-110 ; 5-v_{s_{0}}=0, f_{M} \neq 0, \omega_{m 0}=-228\right)$ и при $m=1,45, \varkappa_{0}=0,65 \mathrm{\kappa r} / \mathrm{\kappa r}$, $\bar{\delta}=67 \cdot 10^{-6}\left(\square-\right.$ данные $\left.\left[{ }^{3}\right]\right) \quad\left(6-\dot{v}_{s 0}=0, f_{M}=0 ; 7-v_{s 0}=0, f_{M} \neq 0, \omega_{m_{0}}=-70\right)$.

ментов $\left[{ }^{3}\right]$. Начальные поля радиальных и угловых скоростей частиц задавались теоретически в соответствии с представлениями применяемой миграционной модели, а распределение вязкости чистого газа $\varepsilon$ заимствовалось из $\left[{ }^{4}\right]$. При описании дисперсной части двухфазного потока использовался общепринятый подход - подход сплошности [5]. Замыкание системы уравнений двухфазной турбулентной струи осуществлялось однопараметрической моделью А. Н. Секундова для чистого газа $\left[{ }^{6}\right]$ (решается система уравнений чистой струи $\left.(1)-(3)\right)$, а для связи характеристик двухфазного течения (турбулентной вязкости газовой фазы $\varepsilon_{g}$ и кажущейся турбулентной вязкости дисперсной фазы $\varepsilon_{s}$ ) с характеристиками чистого газа (турбулентной вязкостью чистого газа $\varepsilon$ ) использовались соотношения модели Г. Н. Абрамовича |[ $\left.{ }^{2}\right]$. Из соотношений последней гипотезы равенства длин путей смешения по концентрации и скорости газовой фазы, т. е. равенства единицы аналога Шмидта $S c$ в двухфазных потоках, которое подтверждается исследованиями $\left[{ }^{7,8}\right]$, получается выражение для коэффициента турбулентной диффузии примеси $D_{s}$. Тогда с учетом вышеизложенного можно написать:

$$
\begin{gathered}
\varepsilon_{g}=\left(\frac{1+n \bar{\alpha}+\left(v_{p 0} / v_{0}^{\prime}\right) \bar{\alpha}}{1+\bar{\alpha}}\right) \varepsilon \\
\varepsilon_{s}=\frac{1}{2}\left(\frac{1-n+\left(v_{p 0} / v_{0}^{\prime}\right) \bar{\alpha}}{1+\bar{\alpha}}\right)\left(\frac{1-n+\left(v_{p 0} / v_{0}^{\prime}\right) \bar{\alpha}}{1+\bar{\alpha}}+\frac{v_{p 0}}{v_{0}^{\prime}}\right) \varepsilon
\end{gathered}
$$




$$
D_{s}=\left(\frac{1+n \bar{\alpha}+\left(v_{p 0} / v_{0}^{\prime}\right) \bar{\alpha}}{1+\bar{\alpha}}\right)\left(\frac{1-n+\left(v_{p 0} / v_{0}^{\prime}\right) \bar{\alpha}}{1+\bar{\alpha}}\right) \varepsilon
$$

Величина $n$, входящая в эти соотношения, характеризует увлекаемость частиц турбулентным газовым молем и рассчитывается по $\left[{ }^{2}\right]$, а $v_{p_{0}}$ и $v_{0}^{\prime}$ характеризуют начальную пульсационную скорость частиц и пульсационную скорость чистого турбулентного образования. Для определения $v_{p_{0}}$ можно воспользоваться соотношениями $\left[{ }^{9}\right]$, либо получить уравнение, схожее с обычным дифференциальным уравнением для величины $n$, предложенное в $\left[{ }^{2}\right]$. Нами использовался последний подход. Среднее значение концентрации $\bar{\alpha}$ берется как среднеинтегральное по каждому сечению струи.

Входящие в уравнение переноса импульса газовой фазы (5) и уравнение переноса импульса дисперсной фазы (7) в осевом направлении, а также в уравнение переноса импульса дисперсной фазы в радиальном направлении (8) члены с коэффициентами $f_{D}$ и $f_{M}$ определяют влияние сил межфазового взаимодействия в осредненном движении - сил сопротивления и Магнуса соответственно. Коэффициенты $f_{D}, f_{M}$, а также коэффициент $f_{\omega}$ в уравнении переноса момента импульса составляющих дисперсной фазы (9) характеризуют рслаксационные свойства движения примеси твердых частиц под действием сил сопротивления, Магнуса и затухания момента вращения частиц из-за ламинарной вязкости $v$. Выражения для коэффициентов $f_{D}, f_{M}$ и $f_{\omega}$ (безразмерная форма) имеют вид:

$$
\begin{gathered}
f_{D}=\frac{3}{8} \frac{\varrho}{\varrho p} c_{D} \frac{\sqrt{\left(u_{g}-u_{s}\right)^{2}+\left(v_{g}-v_{s}\right)^{2}}}{\delta_{1}}, \\
f_{M}=3 \varrho \omega /(4 \varrho p), \\
f_{\omega}=30 \varrho k /\left(\varrho p \delta_{1}^{2} \mathrm{Re}\right) .
\end{gathered}
$$

Здесь $\varrho$ и $\varrho p-$ физические плотности газовой фазы (воздуха) и материала частиц, Re - число Рейнольдса несушей фазы. Коэффициент сопротивления $c_{D}$ рассчитывался по $\left[{ }^{10}\right]$. Коэффициент $k$ характеризует отклонение формы частиц от сферической. Для бронзовых сферических частиц $k$ принимался равным единице. Относительный диаметр частиц $\delta_{1}=\delta / D$.

Система уравнений (1)-(9) в отличие от существующих ныне систем уравнений, описывающих двухфазные турбулентные струи $\left[{ }^{7,11,12}\right]$ содержит два новых уравнения (8) и (9), которые необходимы для выявления силы Магнуса (члены в уравнениях движения с коэффициентом $\left.f_{M}\right)$.

Численная реализация системы уравнений (1)-(9) осуществлялась методом $\left[{ }^{13}\right]$, базирующимся на $\left[{ }^{14}\right]$. Шаблон записывался в неявном виде и вычислялся методом прогонки. Монотонная аппроксимация искомой функции достигалась благодаря оригинальной записи свободных членов, предложенной А. А. Самарским, а использование противопоточной схемы позволяло давать решения для струй (затопленных) практически с любой асимптотически малой спутностью, правда, при этом ухудшалась точность решения (уравнения с первым порядком точности). Совместное решение уравнений (1) и (2) позволяет определить в квадратурах выражение для радиальной составляющей скорости чистого газа, которое решается при вычислении интеграла методом Симпсона. Выражение для радиальной составляющей скорости газовой фазы аналогично по форме уравнению переноса импульса чистого газа в радиальном направлении и отличается от него дополнительным интегралом 
от суммарного воздействия сил сспротнвления и Магнуса. Точность решения системы уравнений (1)-(9) проверялась интегральными законами сохранения суммарного импульса и массы примеси и составляла не менее $5-7 \%$.

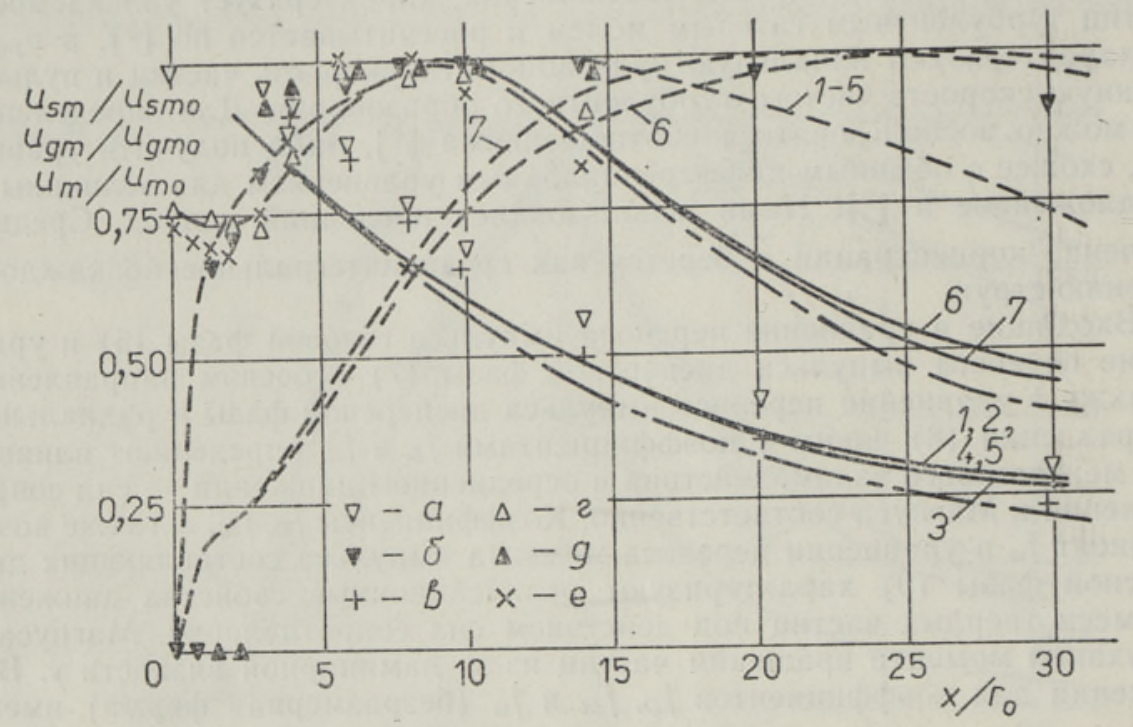

Рис. 2. Осевое распределение скоростей газовой и дисперсной фазы в двухфазной струе и чистой струи (расчетное - сплошные, штриховые и штрихпунктирные кривые соответственно для газовой, дисперсной и беспримесной фаз и экспериментальное $(a-e)$ при $m=0,47, \varkappa_{0}=0,85 \mathrm{\kappa r} / \mathrm{kг}, \bar{\delta}=45 \cdot 10^{-6}$ м $(a-8)$ и при $m=1,45, \quad x_{0}=0,65 \quad \mathrm{\kappa г} / \mathrm{\kappa г}$ $\bar{\delta}=67 \cdot 10^{-6}$ м $\left.(2-e)\right)$. Обозначения $1-7$ см. на рис. 1 .

Результаты решения системы уравнений (1)-(9) представлены на рис. $1,2,3$. Как показали экспериментальные исследования $\left[{ }^{3}\right]$, примесь достаточно интенсивно начинает мигрировать в ядро двухфазной спутной струи, при этом на расстоянии порядка трех калибров концентрирование частиц (рост $\alpha$ ) в приосевой зоне уже завершается.

Экспериментальный материал описывался при помощи двух моделей: турбулентно-диффузионной (отсутствует сила Магнуса, кривые 1, 2, 6 на рис. 1) и миграционной (кривые $3-5,7$ на рис. 1). Одним из необходимых элементов миграционной модели является угловая скорость в начальном сечении струи. При движении твердой примеси в круглых нли коаксиальных трубах частицы трутся об их поверхность, приобретая при этом радиальный и вращательный импульс, поэтому существует возможность распределения ненулевых начальных значений $v_{s}$ и венным подтверждением столкновительного движения частиц в трубах являются экспериментально обнаруженное заметное начальное скольжение фаз (разность осевых скоростей частиц и газа) в каналах $\left[{ }^{15}\right]$ и аномальное распределение примеси в виде эффектов шнурования и разбрасывания в струе $\left[{ }^{16}\right]$. Их возможно объяснить лишь с точки зрения миграционного движения под действием силы Магнуса и начального радиального движения дискретной фазы. Сравнение осевого затухания концентрации, рассчитанной по турбулентно-диффузионной модели, с концентрацией, рассчитанной по миграционной модели (рис. 1), указывает на необходимость использования последней при описании столь интенсивных процессов рассеивания. Особенно это относится к струям со спутностью $m=0,47$ (кривые $1-5$ на рис. 1). Вполне удовлетворительное описание скоростных полей фаз (газовой и дисперсной) в обоих 
случаях спутности дает миграционная модель (рис. 2). Сопоставление распределения скорости газовой фазы в двухфазной спутной и в аналогичной чистой струе в начальных участках струи $x<5 D$ подтверждает экспериментальный и теоретический выводы о том, что примесь слабо влияет на формирование полей однофазной струи, распространяющейся в двухфазном спутном потоке. Дальше вниз по течению $x \geqslant 5 D$, как показывают радиальные распределения скоростей обеих фаз и массовой концентрации (сечение $x=5 D$ на рис. 3 ), начальные особенности полей вырождаются, и двухфазная спутная струя распространяется по законам двухфазной затопленной струи. При этом растет влияние примеси на осредненные параметры, струя становится дальнобойней и у́же. Это видно из сравнения сплошных кривых $1-5$ и 6,7 со штрихпунктирными на рис. 2.

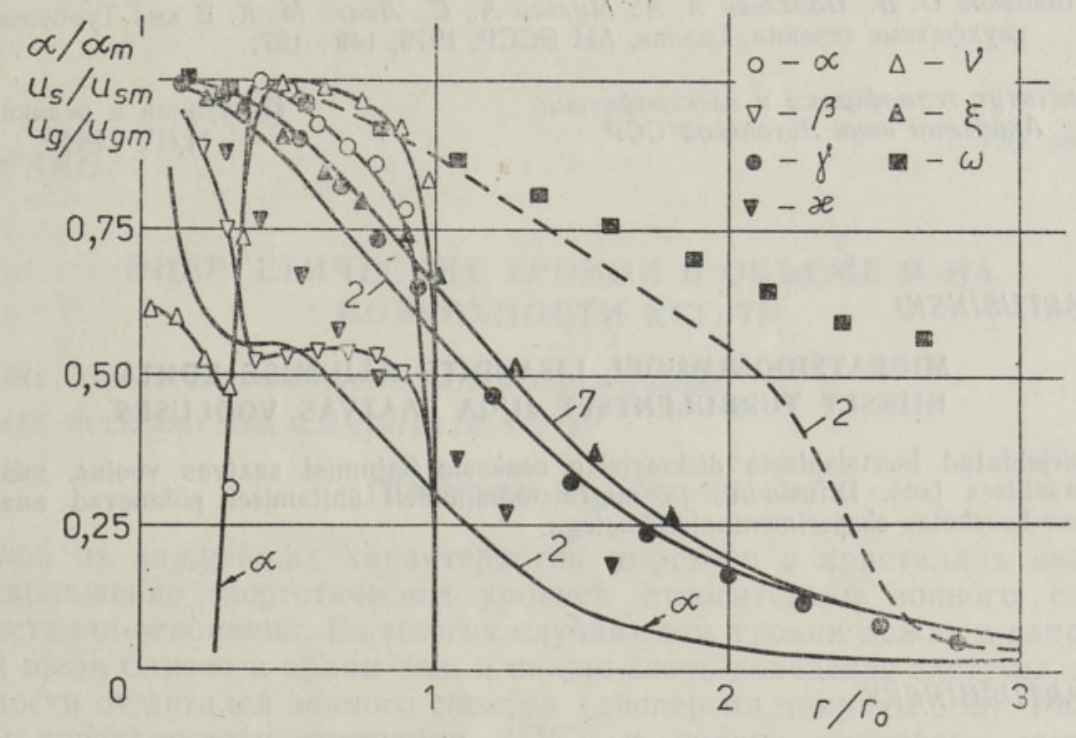

Рис. 3. Радиальное распределение концентрации примеси и скоростей фаз при двух параметрах спутности: при $m=0,47, x_{0}=0,85 \mathrm{kг} / \mathrm{kr}, \bar{\delta}=45 \cdot 10^{-6}$ м $(x=0, \alpha-$ концентрация, $\beta$ - газовая фаза; $x=10 r_{0}, \gamma-$ концентрация, $x-$ газовая фаза, $\omega-$ дисперсная фаза) и при $m=1,45, x_{0}=0,65 \mathrm{kr} / \mathrm{kr}, \bar{\delta}=67 \cdot 10^{-6}$ м $(x=0, v$ - газовая фаза; $x=10 r_{0}, \zeta-$ газовая фаза). Обозначения 2, 7 см. на рис. 1 .

В целом, полученные результаты расчета двухфазной турбулентной спутной струи по миграционной модели, как и в случае двухфазной турбулентной затопленной струи, подтверждают универсальность данной модели. Однако строгое решение задачи о распространении двухфазной спутной турбулентной струи требует предварительного решения задачи о трубном движении твердой примеси, которая к настоящему времени не решена.

\section{ЛИТЕРАТУРА}

1. Абрамович Г. Н., Бажанов В. И., Гиршович Т. А. В кн.: Турбулентные двухфазные течения. Таллин, АН ЭССР, 1976, 47-68.

2. Абрамович Г. Н. Докл. АН СССР, 190, № 5, 1052-1055 (1970).

3. Гириович Т. А., Картушинский А. И., Леонов В. А., Мульги А. С. Изв. АН ЭССР. Физ. Матем., 32, № 3, 327-329 (1983).

4. Хинце И. О. Турбулентность. М., «Физматгиз», 1963. 
5. Нигматулин Р. И. Основы механики гетерогенных сред. М., «Н̈аука», 1978.

6. Секундов А. Н. Изв. АН СССР. Мех. жидкости и газа, № 5, 114-127 (1971).

7. Melville, W. K., Bray, K. N. C. Int. J. Heat and Mass Transfer, 22, 647-656 (1979).

8. Зуев Ю. В., Лепешинский И. А. Изв. АН СССР. Мех. жидкости и газа, № 6, 70-77 (1981).

9. Абрамович $Г$. Н., Гириович T. А. Изв. АН СССР. Мех. жидкости и газа, № 4, $18-23$ (1975).

10. Картушинский А. И., Фришман Ф. А. В кн.: Струйные течения жидкостей и газов. Тез. Всесоюз. науч. конф., III. Новополоцк, НПИ, 1982, 22-28.

11. Васильков А. П. Изв. АН СССР. Мех. жидкости и газа, №. 5, 57-63 (1976).

12. Гавин Л. В., Наумов В. А., Никулин Н. М. Изв. АН СССР. Мех. жидкости и газа, № 1, 48-53 (1983).

13. Кузнецов В. Р., Лебедев А. Б., Секундов А. Н., Смирнова И. П. Изв. АН СССР. Мех. жидкости и газа, № 1, 31-40 (1977).

14. Самарский А. А. Введение в теорию разностных схем. М., «Наука», 1971.

15. Лаатс М. К., Мульги А. С. В кн.: Турбулентные двухфзаные течения. Таллин, АН ЭССР, 1979, 32-46.

16. Навознов О. И., Павельев А. А., Мульги А., С., Лаатс М. К. В кн.: Турбулентные двухфазные течения. Таллин, АН ЭССР, 1979, 149-157.

Ннститут термофизики и электрофизики Академии наук Эстонской ССР

\section{Поступила в редакцию} 11/IV 1984

\section{A. KARTUSINSKI}

\section{MIGRATSIOONIMUDEL LISANDITE HAJUMISE KOHTA HIIBSET TURBULENTSET JUGA SAATVAS VOOLUSES}

On kirjeldatud inertsiaalsete diskreetsete osakeste hajumist saatvas voolus, mis levib kahefaasilises jaos. Difusiooni- ja migratsioonimudeli ühitamisel põhinevad analüüsid on heas kooskōlas eksperimentaalandmetega.

\section{A. KARTUSHINSKY}

\section{THE MIGRATION MODEL OF DIFFUSION OF ADMIXTURE FOR THE TWO-PHASE TURBULENT JET IN A GENERAL STREAM}

The theoretical analysis of the diffusion process of solid admixture for the two-phase jet in a general stream with two parameters of the general stream $(m=0.47$ and $m=1.45$ ) is presented by the numerical method.

The Magnus force which plays an important role in developing the concentration fields of admixture is, according to our calculations, on the order of the turbulent diffusion input. In the equation system, the Magnus force is described by the momentum equation in the radial direction, whereas the moment of the momentum equation is described in the dispersed phase. The advantage of the accepted migration model in comparison with the turbulent diffusion model has been shown. The equations of the pure gas jet were closed by one-parameter turbulence model. The approximation of the velocity correlation between the two phases (gas-solid admixture) in a pulsating motion is based on the Abramovich suggestion. Besides, to.h the drag force and Magnus force act on gas and solid phases in a mean flow. 